\title{
Coronavirus Vaccine: Light at the End of the Tunnel
}

\author{
Krishna M ELLa AND V Krishna MOHAN \\ Bharat Biotech International Limited, Genome Valley, Shamirpet, Hyderabad, Telangana, India. \\ Address for correspondence: VKrishna Mohan, Executive Director, Bharat Biotech International Limited, Genome Valley, \\ Shamirpet, Hyderabad, Telangana, India.kmohan@bharatbiotech.com
}

The world is currently facing an unprecedented global pandemic caused by Severe Acute Respiratory Syndrome Coronavirus 2 (SARS-CoV-2). Predicting the next source of the pandemic can be very challenging. As vaccination is the best way to prevent an infectious disease, the development of an effective vaccine against SARS-CoV-2 can not only reduce the morbidity and mortality associated with it, but can also lessen the economic impact. As the traditional method of vaccine development takes many years for a vaccine to be available to the society, the vaccine development for SARS-CoV-2 should be speeded up using a pandemic approach with fast-track approvals from the regulatory authorities. Various challenges associated with developing a vaccine during the pandemic such as technological hurdles, clinical development pathways, regulatory issues, and support from global funding agencies are expressed here.

Keywords: COVID-19, Immunization, Pandemic, Prevention, SARS-CoV-2.

Published online: April 15, 2020; PII: S097475591600163

$\mathrm{M}$ any cases of respiratory illness started appearing in the month of December, 2019 in Hubei Province, China [1]. The microorganism responsible for this illness was subsequently discovered as coronavirus, and later categorized as genetically related to coronavirus (SARS$\mathrm{CoV}$ ) that was responsible for the severe acute respiratory syndrome (SARS) which occurred in 2003. This new virus was named as Severe Acute Respiratory Syndrome Coronavirus 2 (SARS-CoV-2) and the disease was designated as Coronavirus disease 2019 (COVID-19) by World Health Organization (WHO) [2,3].

Structural analyses have shown that this novel virus uses host cell receptor known as AngiotensinConverting Enzyme-2 for its binding [4]. Transmission dynamics confirmed that reproductive number $(\mathrm{R} 0$, which signifies the number of people who can get infected from one contagious person) of SARS-CoV-2 can be in the range of 2-3, and thus has the potential to spread rapidly [5]. Based on the clinical and epidemiological data from the Chinese health authorities, clinical manifestations of the disease by severity have shown that mild cases occur in $81 \%$ of cases, severe disease is seen in $14 \%$ of cases and critical disease has occurred in 5\% of cases [6]. Considering the infectivity and the severity of COVID-19, vaccines and therapeutics to tackle this deadly disease are the greatest need of the hour.

\section{CAN WE PREDICT THE NEXT PANDEMIC?}

Global pandemic is a major public health concern. Modern means of travel and the volume of travel make it easier for any virus to rapidly spread across the world. Pandemic is a once-in-a-lifetime low-probability event but a high-cost problem that should not be ignored. The next pandemic was anticipated to be a more virulent form of influenza but the world is currently in the midst of a novel coronavirus pandemic.

Though Middle East Respiratory Syndrome (MERS) and SARS were featured in the WHO's list of critical infectious diseases in 2018, it was not anticipated to cause a global disease within a short span of time [7]. Such is the unpredictability of a global pandemic that the world is currently facing.

\section{THE NEED FOR A COVID-19 VACCINE}

As the infectivity of the SARS-CoV-2 virus is very high compared to other corona viruses reported so far, an effective vaccine is the best way to contain the rapidly escalating proliferation of this infection. Although the mortality rate is lower compared to other similar viral respiratory diseases such as Middle East Respiratory Syndrome Coronavirus (MERS-CoV), the magnitude of the infection caused by SARS-CoV-2 is severe and higher, to a large extent, leading to increased number of deaths worldwide.

SARS-CoV-2 infection has the potential to become a seasonal disease like influenza and persist with humanity in the future [8]. An effective vaccine can help in reducing the rate of infection, and can significantly reduce the morbidity and mortality of COVID-19. Vaccine can also 
decrease the probability of resurgence of the disease and its future impact. The implementation of an effective vaccination is the only way to moderate the economic burden of this unprecedented pandemic.

\section{SPEEDING-UP VACCINE DEVELOPMENT DURING PANDEMIC}

There is an urgent need to expedite the development of COVID-19 vaccine. The vaccine industry was able to develop H1N1 vaccine fairly rapidly because the technology and regulations were already in place to develop influenza vaccine. Global experiences with influenza vaccines have outlined the urgency for investing in modern technologies for faster vaccine development as well as increasing the scale of production. Customizing these technologies to various other viruses can speed up vaccine development during a pandemic.

Organizations such as Coalition for Epidemic Preparedness Innovation (CEPI), an innovative global partnership between public and private organizations are striving to quicken vaccine development for various potential and critical diseases, as well as facilitate uniform availability of these life-saving vaccines. CEPI aims to develop vaccines for various pathogens till phase $2 \mathrm{a}$ stage, which can be expedited to full-scale development during future outbreaks [9].

CEPI and the World Bank organized a conference on the three main imperatives for COVID-19 vaccine effort: speed, manufacture and deployment at scale, and global access, which has ultimately led to the formulation of COVID-19 Vaccine Development Taskforce [10].

\section{Multiple Technology Platforms Under Evaluation}

The wide ranges of technologies that are being developed for SARS-CoV-2 include nucleic acids, protein subunit, replicating viral vector, non-replicating viral vector, and inactivated virus approaches. Newer approaches based on nucleic acids such as DNA or mRNA can facilitate potentially rapid production, as they do not need fermentation [9]. For some platforms, adjuvants could improve the immune responses with lower doses thus ensuring vaccination of larger populations without any reduction in efficacy [11]. Currently, several platforms are being developed and the list of SARS-CoV-2 vaccine candidates in development is given in Web Table 1.

\section{CHALLENGES IN COVID-19 VACCINE DEVELOPMENT}

The development of a vaccine for SARS-CoV-2 can cause distinct challenges.
- Developing a viable immunogen using the various proteins of SARS-CoV-2 such as S protein, N protein, $\mathrm{M}$ protein is the initial challenge.

- Development of successful animal model for COVID19 may be challenging although two animal models; one hACE2 transgenic mice model and another, primate macaques model have been successfully developed. This may be due to the highly infectious and pathogenic nature of the virus [12].

- Vaccine development is a lengthy process, starting from product development to the competition of the phase III clinical trial before marketing the vaccine, which can take several years, usually 10 to 20 years.

- Preclinical experience with other SARS vaccine candidates has created red flags about worsening of the disease, which may be attributed to antibodydependent enhancement.

- Correlates of protection are not known.

- Planning and coordinating clinical trials in these emergency-like situations can be difficult, both for predicting the trial sites for outbreak as well as ensuring the site's preparedness.

- In a high-mortality situation such as COVID-19 pandemic, regulators may not accept conventionally designed clinical trials such as comparison with a placebo arm.

- Viral mutation may lead to different subtypes thus causing difficulty in vaccine design.

- Vaccines are generally manufactured using Good Manufacturing Practices (GMP) to ensure that quality is controlled and consistency is maintained. For many vaccine candidates in the current pipeline for SARS-CoV-2, these GMP processes need to be developed, which can be time consuming.

- For vaccines that will be developed with novel technologies, GMP needs to be developed from the beginning, thus adding to the financial burden and delaying the production of vaccines.

- Issues related to vaccine ownership, funding, pricing and supply chain, and the coordinated administration strategy can pose significant barriers.

\section{Role of Regulatory Bodies}

The traditional vaccine development process follows various phases such as product development, preclinical, clinical trial phases (phase I, II, and III) before the vaccine gets regulatory approval for marketing although phase IV and post-marketing surveillance studies are 
conducted after marketing the product. This traditional approach is time consuming and not feasible to follow during the pandemic.

Keeping greater benefit of the vaccine for the societal need, vaccine development using pandemic approach will be the best approach for rapid development of vaccines as shown in Fig. 1. This type of innovative approach can save the time spent in pre-clinical and clinical trial phases. The regulators should consider this approach for faster approval of vaccines.

\section{Funding for Vaccine Development During Pandemics}

Vaccine development during a pandemic should be considered as a global health emergency and not as any specific disease related issue, which is a prerogative of vaccine manufacturers. Public funding for vaccine development should be the top priority as any vaccine development, particularly during a pandemic can be a very risky investment, and public funding can reduce the potential risk for vaccine manufacturers, especially in the low and middle income countries.

Pioneering finance mechanisms such as the International Finance Facility for Immunization (IFFIm) that have been successful in the past should be used to fund the development of COVID-19 vaccines [10]. Global Alliance for Vaccines and Immunizations (GAVI) board members also expressed support for the use of GAVI's innovative financing instruments, such as its IFFIm and GAVI's Advance Market Commitment to accelerate vaccine development and access where needed [13].

There should be a global consensus and urgency for the development of a pandemic vaccine, at both national and international level to ensure that the vaccines are available and affordable to those who need them the most [14].

\section{Challenges After the Vaccine is Developed}

- For novel platform technologies, large-scale manufacturing can pose a significant financial risk as these facilities necessitate huge investments.

- High-income countries can procure large doses of vaccine for their own population, creating disparities in the global supply and demand.

- High risk populations should be identified through epidemiological and serological studies and vaccine delivery should be prioritized. This can halt the spread of the disease.

- As SARS-CoV-2 is a newer disease without adequate exposure in the population, any new vaccine may probably necessitate more than one dose of the vaccine and protective immunity is usually achieved after the second dose. Massive effort will be needed to ensure vaccine distribution and administration.

- There can be a scenario, albeit low-probability, where the pandemic threat has been abruptly curtailed. In such cases, vaccine development should be continued for potential vaccine candidates to restrict future threats.
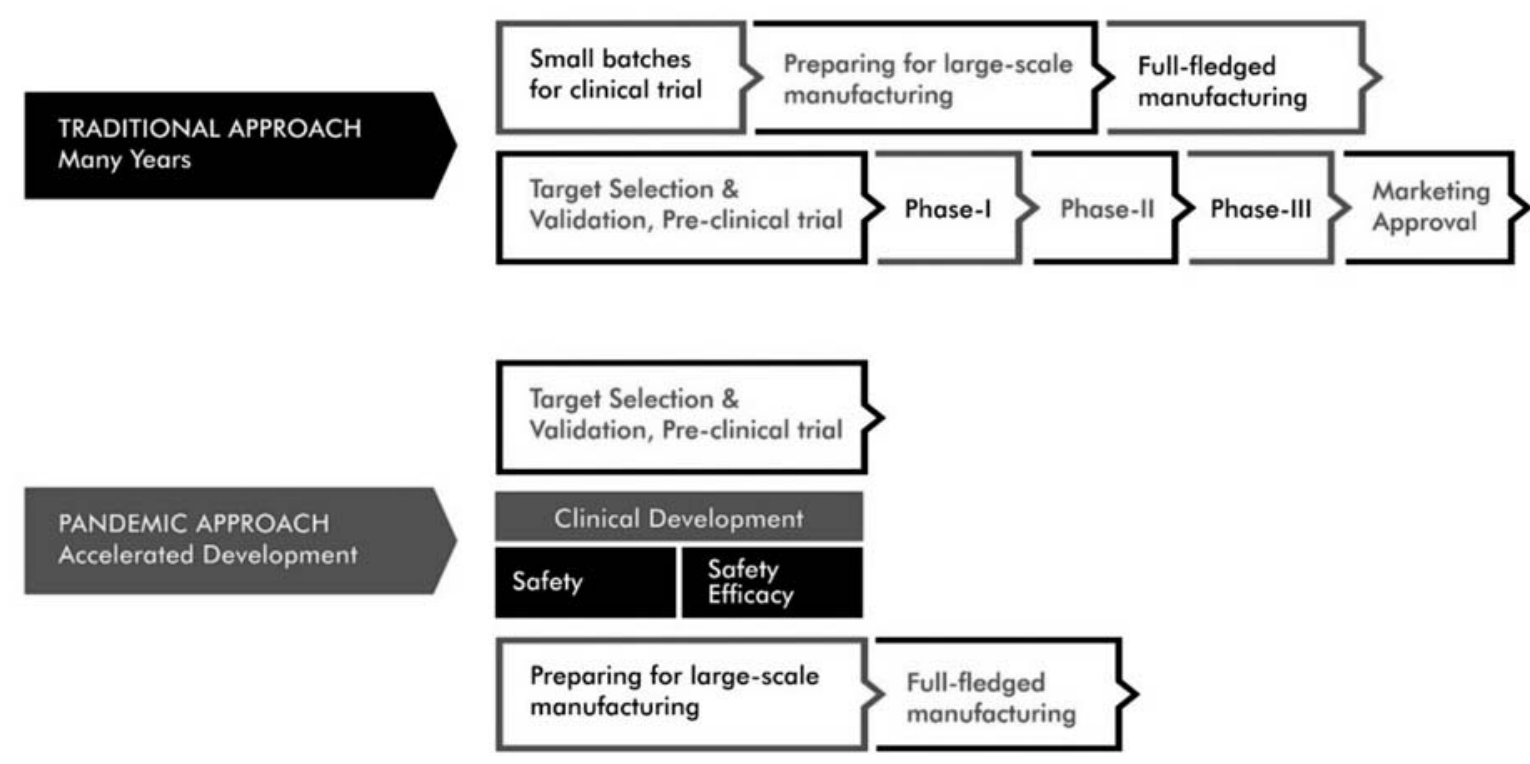

Fig. 1 Traditional approach vs. pandemic approach of vaccine development. 


\section{FUTURE OUTLOOK}

The global vaccine development efforts for COVID-19 pandemic are unparalleled. In the current scenario, there is an indication that vaccine could be available as early as 2021. This would be an extraordinary shift from the usual vaccine development timeframe which can range between 10-20 years. A new virus target and newer technologies can multiply the vaccine development risks, and dictates meticulous evaluation of safety and efficacy at every stage of vaccine development.

Robust coordination between vaccine manufacturers, regulatory authorities, public health authorities and governments is essential to make sure that potential vaccine candidates are fast-tracked for approval, manufactured and distributed seamlessly, particularly for low-income countries.

Considering the economic impact that COVID-19 has already caused in the initial 3-4 months of this global calamity, it is worthwhile to start investing in vaccines against emerging viruses, including neglected diseases, which can potentially cause significant human deaths and also impact the global economy. Global pandemics are inevitable. There should be strategically prepared protocols and emergency plans to develop vaccines in months rather than years. Lessons learned in managing the COVID-19 pandemic should pave the way for creating better roadmaps to face the next pandemic.

Acknowledgements: Shashi Kanth Muni (Associate Medical Director, Bharat Biotech) and Dr. Sapan Kumar Behera (Senior Manager, Bharat Biotech) supported the preparation of this manuscript.

Funding: None; Competing interests: KME is Chairman and Managing Director and VKM is Executive Director of Bharat Biotech International Limited, which is a vaccine manufacturer.

\section{REFERENCES}

1. Huang C, Wang Y, Li X, Ren L, Zhao J, Hu Y, et al. Clinical features of patients infected with 2019 novel coronavirus in Wuhan, China. Lancet. 2020;395:497-506.

2. Lu R, Zhao X, Li J, Niu P, Yang B, Wu H, et al. Genomic characterisation and epidemiology of 2019 novel coronavirus: implications for virus origins and receptor binding. Lancet.2020;395:565-74.

3. Gorbalenya AE, Baker SC, Baric RS, de Groot RJ, Drosten $\mathrm{C}$, Gulyaeva AA, et al. Severe acute respiratory syndromerelated coronavirus: the species and its viruses - a statement of the coronavirus study group. BioRxiv. 2020;20200207: 937862 .
4. Wan Y, Shang J, Graham R, Baric RS, Li F. Receptor recognition by novel coronavirus from Wuhan: An analysis based on decade-long structural studies of SARS. J Virol. 2020.Available from: $h t t p s: / / j v i . a s m . o r g / c o n t e n t / j v i / 94 / 7 /$ e00127-20.full.pdf. Accessed April 10, 2020.

5. Li Q, Guan X, Wu P, Wang X, Zhou L, Tong Y, et al. Early transmission dynamics in Wuhan, China, of novel coronavirus-infected pneumonia. $\mathrm{N}$ Engl J Med. 2020.Available from: https://www.nejm.org/doi/full/ 10.1056/NEJMoa2001316.Accessed April 11, 2020.

6. Cascella M, Rajnik M, CuomoA,Dulebohn SC, Di Napoli R. Features, evaluation and treatment coronavirus (COVID-19). Available from:https://www.ncbi.nlm.nih. gov/books/NBK554776/.Accessed April 11, 2020.

7. WHO 2018 Annual review of diseases prioritized under the Research and Development Blueprint. https://www.who.int/ blueprint/priority-diseases/key-action/novel-coronaviruslandscape-ncov.pdf. Accessed April 11, 2020.

8. Zhang J, Zeng H, Gu J, Li H, Zheng L, Zou Q. Progress and prospects on vaccine development against SARS-CoV2. Vaccines (Basel). 2020;8:153. Available from:https:// www.mdpi.com/2076-393X/8/2/153/htm.Accessed April $10,2020$.

9. Lurie N, Saville M, Hatchett R, Halton J. Developing Covid-19 vaccines at pandemic speed. N Engl J Med. 2020 Mar 30. [Epub ahead of print]. Available from: https:// www.nejm.org/doi/full/10.1056/NEJMp2005630. Accessed April 12, 2020.

10. Yamey G, Schäferhoff M, Hatchett R, Pate M, Zhao M, McDade KK. Ensuring global access to COVID-19 vaccines. Lancet. 2020.Available from: https:// www.thelancet.com/journals/lancet/article/PIISO1406736(20)30763-7/fulltext.Accessed April 11, 2020.

11. Le TT, Andreadakis Z, Kumar A, Román RG, Tollefsen S. The COVID-19 vaccine development landscape. Nature Rev Drug Disc. 2020.Available from: https:// www.nature.com/articles/d41573-020-00073-5. Accessed April 11, 2020.

12. Dhama K, Sharun K, Tiwari R, Dadar M, Malik YS, Singh KP. COVID-19, an emerging coronavirus infection: Advances and prospects in designing and developing vaccines, immunotherapeutics, and therapeutics. Hum Vaccin Immunother. 2020 Mar 18 [Epub ahead of print]. Available from: https://www.tandfonline.com/doi/full/ 10.1080/ 21645515.2020.1735227. Accessed April 12, 2020.

13. Global Alliance for Vaccines and Immunisation (Gavi.org) Media room.Available from:https://www.gavi.org/news/ media-room/gavi-board-calls-bold-engagement-respondcovid-19. Accessed April 12, 2020.

14. Fedson DS. Vaccine development for an imminent pandemic: Why we should worry, what we must do. Human Vacc. 2006;2:38-42. 